\title{
Copper(I) complexes with 3-(allylamino)-, 3-(diallylamino)-, 3-[(2-hydroxyethyl)(allyl)amino]- and 3-[(2-hydroxyethyl)amino]- propanenitriles. Synthesis and crystal structure of $\left[\left\{\mathrm{C}_{3} \mathrm{H}_{5} \mathrm{NH}\left(\mathrm{H}^{+}\right) \mathrm{C}_{2} \mathrm{H}_{4} \mathrm{CN}\right\} \mathrm{Cu}_{2} \mathrm{Br}_{3}\right]$, $\left[\left\{\left(\mathrm{C}_{3} \mathrm{H}_{5}\right)_{2} \mathrm{~N}\left(\mathrm{H}^{+}\right) \mathrm{C}_{2} \mathrm{H}_{4} \mathrm{CN}\right\} \mathrm{CuCl}_{1.94} \mathrm{Br}_{0.06}\right]$, $\left[\left\{\mathrm{HOC}_{2} \mathrm{H}_{4} \mathrm{NH}\left(\mathrm{H}^{+}\right) \mathrm{C}_{2} \mathrm{H}_{4} \mathrm{CN}\right\} \mathrm{CuCl}_{2}\right]$ and $\left[\left\{\mathrm{HOC}_{2} \mathrm{H}_{4}\left(\mathrm{C}_{3} \mathrm{H}_{5}\right) \mathrm{N}\left(\mathrm{H}^{+}\right) \mathrm{C}_{2} \mathrm{H}_{4} \mathrm{CN}\right\} \mathrm{CuCl}_{2}\right]$
}

\author{
Mykhaylo LUK'YANOV ${ }^{1 *}$, Vasyl KINZYBALO ${ }^{2}$, Evgeny GORESHNIK ${ }^{3}$, Oleksiy PAVLYUK ${ }^{1}$, \\ Maryan MYS'KIV ${ }^{1}$ \\ ${ }^{1}$ Department of Inorganic Chemistry, Ivan Franko National University of Lviv, \\ Kyryla i Mefodiya St. 6, 79005 Lviv, Ukraine \\ ${ }^{2}$ Faculty of Chemistry, University of Wroclaw, F. Joliot-Curie 14, 50-383 Wroclaw, Poland \\ ${ }^{3}$ Department of Inorganic Chemistry and Technology, Jožef Stefan Institute, \\ Jamova 39, SI-1000 Ljubljana, Slovenia \\ * Corresponding author.Tel.: +380-32-2394-506; e-mail: mishalukianov@gmail.com
}

Received May 18, 2012; accepted June 27, 2012; available on-line November 5, 2012

Four crystalline copper(I) complexes with $\mathrm{N}$-derivatives of propanenitrile: $\left[\left\{\mathrm{C}_{3} \mathrm{H}_{5} \mathrm{NH}\left(\mathrm{H}^{+}\right) \mathrm{C}_{2} \mathrm{H}_{4} \mathrm{CN}\right\} \mathrm{Cu}_{2} \mathrm{Br}_{3}\right](\mathrm{I})$, $\left[\left\{\left(\mathrm{C}_{3} \mathrm{H}_{5}\right)_{2} \mathrm{~N}\left(\mathrm{H}^{+}\right) \mathrm{C}_{2} \mathrm{H}_{4} \mathrm{CN}\right\} \mathrm{CuCl}_{1.94} \mathrm{Br}_{0.06}\right] \quad$ (II), $\quad\left[\left\{\mathrm{HOC}_{2} \mathrm{H}_{4} \mathrm{NH}\left(\mathrm{H}^{+}\right) \mathrm{C}_{2} \mathrm{H}_{4} \mathrm{CN}\right\} \mathrm{CuCl}_{2}\right] \quad$ (III) $\left[\left\{\mathrm{HOC}_{2} \mathrm{H}_{4}\left(\mathrm{C}_{3} \mathrm{H}_{5}\right) \mathrm{N}\left(\mathrm{H}^{+}\right) \mathrm{C}_{2} \mathrm{H}_{4} \mathrm{CN}\right\} \mathrm{CuCl}_{2}\right](\mathrm{IV})$, have been obtained (by the alternating current electrochemical technique from alcohol solutions of the organic ligand and $\mathrm{CuCl}_{2} \cdot 2 \mathrm{H}_{2} \mathrm{O}$ or $\mathrm{CuBr}_{2}$ ) and investigated by $\mathrm{X}$-ray diffraction. Crystals of compound I are triclinic, space group $P-1, Z=2, a=6.5520(7), b=7.8642(8)$, $c=11.2370(12) \AA, \alpha=90.694(6), \beta=94.427(6), \gamma=93.690(6)^{\circ}, V=575.99(10) \AA^{3}$. Crystals of compounds II-IV are monoclinic. For II and IV the space group is $P 2_{1} / c, Z=4$. For II: $a=11.6518(4), b=7.0086(2)$, $c=14.6765(5) \AA, \beta=100.965(13)^{\circ}, V=1176.64(7) \AA^{3} ; I V: a=11.001(3), b=7.017(2), c=14.653(4) \AA$, $\beta=97.19(3)^{\circ}, V=1122.2(5) \AA^{3}$. For III the space group is $P 2 / c, Z=4, a=7.452(2), b=7.642(2)$, $c=15.867(5) \AA, \beta=95.43(3)^{\circ}, V=899.5(4) \AA^{3}$. The structures of the coordination compounds I-IV possess distinct polymer anionic chains $\left(\mathrm{CuX}_{2}\right)_{n}^{n-}$ or $\left(\mathrm{Cu}_{2} \mathbf{X}_{3}\right)_{n}^{n-}(\mathrm{X}=\mathrm{Cl}, \mathbf{B r})$, which are joined together by organic cations $\left(\mathrm{H}^{+} \mathrm{L}\right)(\mathrm{I})$ or surrounded by them (II-IV). Hydrogen bonding play a significant role in the stabilization of the structures.

Copper(I) / $\pi$ - and $\sigma$-Complexes / $\beta$-Aminonitriles / Crystal structure / Electrochemical technique

\section{Introduction}

Aminonitriles form a large class of organic compounds with diverse representatives that find their application in various spheres. More than 150 years have passed since the first synthesis of compounds of this class was mentioned by Strecker [1,2], but the topicality and challenges remain inexhaustible [3] and the chemistry of nitriles is a traditional subject of intensive research in terms of theory and practice. Attempts to systematize nitrile compounds have been made by E.N. Zil'berman [4] but, as mentioned by the author himself, some specific classes have been left untouched since there has never been a substantial basis for a sustained development of a complex methodology [5]. Efforts are underway to expand the scope of efficient methods for the synthesis of these compounds, with growing emphasis on copper(I) compounds with $\mathrm{N}$-derivatives of aminopropanenitrile with further practical potential of explaining the mechanism of interaction of copper(I) salts with aminonitriles [6]. 
The present paper also reports results on new $\mathrm{Cu}(\mathrm{I})$ halide $\pi$-complexes with $\mathrm{N}$-allyl derivatives of aminonitriles.

\section{Experimental section}

\section{Synthesis of 3-(allylamino)propanenitrile $\left(\mathrm{C}_{3} \mathrm{H}_{5} \mathrm{NHC}_{2} \mathrm{H}_{4} \mathrm{CN}, \mathrm{L1}\right)$}

This ligand was obtained from acrylonitrile $(6.8 \mathrm{~mL}$, $0.1 \mathrm{~mol})$ and allylamine $(11.2 \mathrm{~mL}, 0.15 \mathrm{~mol})$. The mixture of reagents was continuously stirred and cooled $\left(5 \mathrm{~h}, 20^{\circ} \mathrm{C}\right)$, maintaining the temperature not higher than $30^{\circ} \mathrm{C}$ [7], then it was heated for $1 \mathrm{~h}$ in a water bath with a reflux condenser at $60^{\circ} \mathrm{C}$. The product (orange liquid) was purified by distillation in vacuum produced by a water-jet pump $\left(85^{\circ} \mathrm{C} /\right.$ $40 \mathrm{~mm} \mathrm{Hg}$ ). The yield of $L 1$ was $88 \%(15 \mathrm{~mL})$.

\section{Synthesis of 3-(diallylamino)propanenitrile $\left(\left(\mathrm{C}_{3} \mathrm{H}_{5}\right)_{2} \mathrm{NC}_{2} \mathrm{H}_{4} \mathrm{CN}, \mathrm{L2}\right)$}

To obtain 3-(diallylamino)propanenitrile a mixture of acrylonitrile $(7 \mathrm{~mL}, 0.11 \mathrm{~mol})$ and diallylamine $(16 \mathrm{~mL}, 0.13 \mathrm{~mol})$ was heated with a reflux condenser in a water bath at $50-60^{\circ} \mathrm{C}$ for $8 \mathrm{~h}$ and then kept at room temperature for $24 \mathrm{~h}$ [7]. A small amount of unreacted initial substances was distilled off in vacuum produced by a water-jet pump; the red-orange liquid was distilled at $130^{\circ} \mathrm{C}(20 \mathrm{~mm} \mathrm{Hg})$. The yield of $L 2$ was $75 \%(13 \mathrm{~mL})$.

\section{Synthesis of \\ 3-(\{2-hydroxyethyl\}amino)propanenitrile \\ $\left(\left(\mathrm{HOC}_{2} \mathrm{H}_{5}\right) \mathrm{NHC}_{2} \mathrm{H}_{4} \mathrm{CN}, L 3\right)$ and \\ 3-(\{2-hydroxyethyl\}allylamino)propanenitrile $\left(\left(\mathrm{HOC}_{2} \mathrm{H}_{5}\right) \mathrm{C}_{3} \mathrm{H}_{5} \mathrm{NC}_{2} \mathrm{H}_{4} \mathrm{CN}, \mathrm{L4}\right)$}

3-(\{2-hydroxyethyl $\}$ amino)propanenitrile (L3) was synthesized from monoethanolamine $(3 \mathrm{~mL}, 0.05 \mathrm{~mol})$ and acrylonitrile $(3.5 \mathrm{~mL}, 0.05 \mathrm{~mol})$. After $24 \mathrm{~h}$ at $25^{\circ} \mathrm{C}$, the mixture was kept at room temperature $\left(19^{\circ} \mathrm{C}\right)$ for $12 \mathrm{~h}$. The resulting colorless liquid was used for further syntheses of $L 4$ and complex III. The yield of $L 3$ was $\sim 75 \%$.

To obtain 3-(\{2-hydroxyethyl $\}$ allylamino)propanenitrile an ethanol-benzene (1:1) solution of $L 3$ and allyl bromide $(1.11 \mathrm{~g}, 0.0128 \mathrm{~mol})$ in the presence of $\mathrm{NaHCO}_{3}(1.465 \mathrm{~g}, 0.0128 \mathrm{~mol})$ was stirred and heated in a reflux condenser $\left(80^{\circ} \mathrm{C}\right)$ for $72 \mathrm{~h}$. Then the mixture was filtered $(\mathrm{NaBr}$ was removed) and the excess of solvents was distilled off under reduced pressure. A yellow liquid was obtained $(17 \mathrm{~mL})$. The yield of $L 4$ was $\sim 90 \%$.

\section{Preparation of $\left(\left[\left(\mathrm{C}_{3} \mathrm{H}_{5} \mathrm{NH}\left(\mathrm{H}^{+}\right) \mathrm{C}_{2} \mathrm{H}_{4} \mathrm{CN}\right) \mathrm{Cu}_{2} \mathrm{Br}_{3}\right]\right.$ (I)}

Colorless needle-like crystals of complex $\mathbf{I}$ appeared from an ethanol $(4 \mathrm{~mL})$ solution of $\mathrm{CuBr}_{2}(1 \mathrm{~g}$,
$4.5 \mathrm{mmol})$ and $L 1(0.6 \mathrm{~mL}, 5 \mathrm{mmol}$, titrated with a concentrated water solution of $48 \% \mathrm{HBr}$ to $\mathrm{pH}=3$ ) under conditions of the alternating current electrochemical technique [8]. The initial solution was placed into a small test-tube and copper-wire electrodes in cork were inserted. After applying $0.65 \mathrm{~V}$ of alternating current (frequency $50 \mathrm{~Hz}$ ) tension for 7 days, crystals of $\mathbf{I}$ were formed on the copper electrodes. The yield of the complex was $70 \%$.

\section{Preparation of \\ $\left.\left[\left(\mathrm{C}_{3} \mathrm{H}_{5}\right)_{2} \mathrm{~N}\left(\mathrm{H}^{+}\right) \mathrm{C}_{2} \mathrm{H}_{4} \mathrm{CN}\right\} \mathrm{CuCl}_{1.94} \mathrm{Br}_{0.06}\right]$ (II)}

Good quality crystals of the mixed halide complex II were obtained in a similar way (alternating current, $U=0.4 \mathrm{~V}, I_{\text {init }}=1 \mathrm{~mA}$, time of synthesis $22 \mathrm{~h}$ ) starting from an ethanol $(4 \mathrm{~mL})$ solution of $0.7 \mathrm{~mL}(4.3 \mathrm{mmol})$ of $L 2$ (titrated with a concentrated water solution of $48 \% \mathrm{HBr}$ to $\mathrm{pH}=4)$ and $0.70 \mathrm{~g}(4.1 \mathrm{mmol})$ of $\mathrm{CuCl}_{2} \cdot 2 \mathrm{H}_{2} \mathrm{O}$. The yield was $50 \%$.

\section{Preparation of $\left[\left(\mathrm{HOC}_{2} \mathrm{H}_{4} \mathrm{NH}\left(\mathrm{H}^{+}\right) \mathrm{C}_{2} \mathrm{H}_{4} \mathrm{CN}\right) \mathrm{CuCl}_{2}\right]$ (III)}

Colorless prismatic crystals of compound III appeared on the copper wire electrodes after $3 \mathrm{~h}(U=0.45 \mathrm{~V}$, $\left.I_{\text {init }}=0.5 \mathrm{~mA}\right)$ from a benzene-ethanol $\left(0.5 \mathrm{~mL} \mathrm{C}_{6} \mathrm{H}_{6}\right.$ and $\left.4.5 \mathrm{~mL} \mathrm{C}_{2} \mathrm{H}_{5} \mathrm{OH}\right)$ solution of $\mathrm{CuCl}_{2} \cdot 2 \mathrm{H}_{2} \mathrm{O}(0.65 \mathrm{~g}$, $3.8 \mathrm{mmol})$ and $L 3(0.68 \mathrm{~mL}, 4 \mathrm{mmol}$, titrated with $36 \% \mathrm{HCl}$ to $\mathrm{pH}=4)$. The yield was $70 \%$.

\section{Preparation of $\left[\left\{\mathrm{HOC}_{2} \mathrm{H}_{4}\left(\mathrm{C}_{3} \mathrm{H}_{5}\right) \mathrm{N}\left(\mathrm{H}^{+}\right) \mathrm{C}_{2} \mathrm{H}_{4} \mathrm{CN}\right\} \mathrm{CuCl}_{2}\right](\mathrm{IV})$}

Crystals of the $\pi$-complex IV grew from a solution of $\mathrm{CuCl}_{2} \cdot 2 \mathrm{H}_{2} \mathrm{O}(0.70 \mathrm{~g}, 4.1 \mathrm{mmol})$ and $L 4(0.72 \mathrm{~mL}$, $4.5 \mathrm{mmol}$, titrated with $36 \% \mathrm{HCl}$ to $\mathrm{pH}=4.5-5)$ in a mixture of $\mathrm{C}_{6} \mathrm{H}_{6}(0.5 \mathrm{~mL}), \mathrm{C}_{2} \mathrm{H}_{5} \mathrm{OH}(2.5 \mathrm{~mL})$ and $\mathrm{C}_{3} \mathrm{H}_{7} \mathrm{OH}(1.5 \mathrm{~mL})$ after $10 \mathrm{~h}$ under conditions of an alternating current electrochemical synthesis $\left(U=0.45 \mathrm{~V}, I_{\text {init }}=0.5 \mathrm{~mA}\right)$. The product yield was $70 \%$.

\section{$X$-ray crystal structure determination}

The crystallographic parameters and summaries of the data collections for I-IV are presented in Table 1. Single crystals of I-IV were preliminarily studied by the photographic method and then diffraction data were collected on a Rigaku AFC7R (for I-II) or KUMA-KM4/CCD (for III-IV) diffractometers (graphite monochromated Mo $K_{\alpha}$ radiation). Lorentz and polarization corrections were applied.

The X-ray data were processed using the Rigaku CrystalClear program [9] for compounds I and II. The CrysAlisRED program was used for processing the X-ray data for complexes III and IV [10]. An absorption correction was applied by the analytical method [11]. 
Table 1 Crystallographic data and experimental details for structures I-IV.

\begin{tabular}{|c|c|c|c|c|}
\hline & I & II & III & IV \\
\hline Empirical formula & $\mathrm{C}_{6} \mathrm{H}_{11} \mathrm{Br}_{3} \mathrm{Cu}_{2} \mathrm{~N}_{2}$ & $\mathrm{C}_{9} \mathrm{H}_{15} \mathrm{Br}_{0.06} \mathrm{Cl}_{1.94} \mathrm{CuN}_{2}$ & $\mathrm{C}_{5} \mathrm{H}_{11} \mathrm{Cl}_{2} \mathrm{CuN}_{2} \mathrm{O}$ & $\mathrm{C}_{8} \mathrm{H}_{15} \mathrm{Cl}_{2} \mathrm{CuN}_{2} \mathrm{O}$ \\
\hline Formula weight & 477.98 & 288.45 & 249.60 & 289.66 \\
\hline Temperature, $\mathrm{K}$ & 200 & 200 & 100 & 100 \\
\hline Wavelength, $\AA$ & $0.71073\left(\right.$ Mo $\left.K_{\alpha}\right)$ & $0.71069\left(\right.$ Мo $\left.K_{\alpha}\right)$ & $0.71073\left(\right.$ Mo $\left.K_{\alpha}\right)$ & $0.71073\left(\right.$ Mo $\left.K_{\alpha}\right)$ \\
\hline $\begin{array}{l}\text { Crystal system, } \\
\text { space group }\end{array}$ & Triclinic, $P-1$ & Monoclinic, $P 2_{1} / \mathrm{c}$ & Monoclinic, $P 2 / \mathrm{c}$ & Monoclinic, $P 2_{1} / \mathrm{c}$ \\
\hline Diffractometer & Rigaku AFC7 & Rigaku AFC7 & KUMA-KM4/CCD & $\begin{array}{l}\text { KUMA- } \\
\text { KM4/CCD }\end{array}$ \\
\hline Unit cell dimensions & & & & \\
\hline$a, \AA$ & $6.5520(7)$ & $11.6518(4)$ & $7.452(2)$ & $11.001(3)$ \\
\hline$b, \AA$ & $7.8642(8)$ & $7.0086(2)$ & $7.642(2)$ & $7.017(2)$ \\
\hline$c, \AA$ & $11.2370(12)$ & $14.6765(5)$ & $15.867(5)$ & $14.653(4)$ \\
\hline$\alpha,^{\circ}$ & $90.694(6)$ & 90 & 90 & 90 \\
\hline$\beta, \circ$ & $94.427(6)$ & $100.965(13)$ & $95.43(3)$ & $97.19(3)$ \\
\hline$\gamma,{ }^{\circ}$ & $93.690(6)$ & 90 & 90 & 90 \\
\hline Volume, $\AA^{3}$ & $575.99(10)$ & $1176.64(7)$ & $899.5(4)$ & $1122.2(5)$ \\
\hline$Z$ & 2 & 4 & 4 & 4 \\
\hline $\begin{array}{l}\text { Calculated density, } \\
\mathrm{g} \cdot \mathrm{cm}^{-3}\end{array}$ & 2.756 & 1.628 & 1.843 & 1.714 \\
\hline $\begin{array}{l}\text { Absorption } \\
\text { coefficient, } \mathrm{mm}^{-1}\end{array}$ & 14.05 & 2.47 & 2.97 & 2.39 \\
\hline$F(000)$ & 448 & 587.6 & 504 & 592 \\
\hline Crystal size, mm & $0.10 \times 0.05 \times 0.04$ & $0.10 \times 0.09 \times 0.03$ & $0.20 \times 0.18 \times 0.16$ & $0.10 \times 0.08 \times 0.05$ \\
\hline Colour, shape & Colorless, prism & Colorless, chunk & Colorless, prism & Colorless, prism \\
\hline $\begin{array}{l}\text { Theta range for data } \\
\text { collection, }\end{array}$ & $1.8-29.0$ & $2.8-28.2$ & $3.0-37.0$ & $2.8-35.1$ \\
\hline Limiting indices & $\begin{array}{c}-8 \leq h \leq 8 \\
-10 \leq k \leq 10 \\
0 \leq l \leq 14\end{array}$ & $\begin{array}{c}0 \leq h \leq 15 \\
-9 \leq k \leq 5 \\
-18 \leq l \leq 18\end{array}$ & $\begin{array}{c}-12 \leq h \leq 12 \\
-12 \leq k \leq 12 \\
-23 \leq l \leq 26\end{array}$ & $\begin{array}{c}-15 \leq h \leq 16 \\
-10 \leq k \leq 10 \\
-23 \leq l \leq 19\end{array}$ \\
\hline Refinement method & $\begin{array}{l}\text { Full-matrix least- } \\
\text { squares on } F^{2}\end{array}$ & $\begin{array}{l}\text { Full-matrix least- } \\
\text { squares on } F^{2}\end{array}$ & $\begin{array}{l}\text { Full-matrix least- } \\
\text { squares on } F^{2}\end{array}$ & $\begin{array}{l}\text { Full-matrix least- } \\
\text { squares on } F^{2}\end{array}$ \\
\hline Measured reflections & 2431 & 3614 & 14333 & 11972 \\
\hline $\begin{array}{l}\text { Independent } \\
\text { reflections }\end{array}$ & 2431 & 2430 & 4030 & 4243 \\
\hline $\begin{array}{l}\text { Observed reflections } \\
{[I>2 \sigma(I)]}\end{array}$ & 1880 & 2301 & 2374 & 3403 \\
\hline Refined parameters & 118 & $\begin{array}{r}189 \\
-2\end{array}$ & 183 & 114 \\
\hline Weighting scheme ${ }^{a}$ & $\begin{array}{c}{\left[\sigma^{2}\left(F_{\mathrm{o}}{ }^{2}\right)+\right.} \\
\left.(0.0355 P)^{2}\right]^{-1}\end{array}$ & $\begin{array}{c}{\left[\sigma^{2}\left(F_{\mathrm{o}}^{2}\right)+\right.} \\
\left.(0.0506 P)^{2}+3.3037 P\right]^{-1}\end{array}$ & $\begin{array}{c}{\left[\sigma^{2}\left(F_{\mathrm{o}}^{2}\right)+\right.} \\
\left.(0.0455 P)^{2}+0.1706 P\right]^{-1}\end{array}$ & $\begin{array}{c}{\left[\sigma^{2}\left(F_{\mathrm{o}}^{2}\right)+\right.} \\
\left.(0.048 P)^{2}\right]^{-1}\end{array}$ \\
\hline $\begin{array}{l}\text { Goodness-of-fit on } \\
\mathrm{F}^{2}\end{array}$ & 0.97 & 1.15 & 1.03 & 1.10 \\
\hline $\begin{array}{l}\text { Final } R \text { indices } \\
{[I>2 \sigma(I)]}\end{array}$ & $\begin{array}{c}R=0.043 \\
w R=0.099\end{array}$ & $\begin{array}{c}R=0.047 \\
w R=0.115\end{array}$ & $\begin{array}{c}R=0.040 \\
w R=0.099\end{array}$ & $\begin{array}{c}R=0.028 \\
w R=0.083\end{array}$ \\
\hline $\begin{array}{l}\text { Residual electron } \\
\text { density, e } \AA^{-3}\end{array}$ & 1.72 and -0.79 & 0.45 and -0.83 & 1.14 and -1.35 & 0.86 and -0.79 \\
\hline
\end{tabular}
${ }^{\mathrm{a}} P=\left(F_{\mathrm{o}}{ }^{2}+2 F_{\mathrm{c}}{ }^{2}\right) / 3$.

Structures I-IV were solved by direct methods and the light atoms were revealed from difference Fourier syntheses, using the SHELX program package [12]. Full-matrix least-squares refinements based on $F^{2}$ were carried out for the positional and thermal parameters of all non-hydrogen atoms. The hydrogen atoms in structures I-III were revealed from difference Fourier syntheses and refined in the riding model along with the non-hydrogen atoms (fixed $\mathrm{C}-\mathrm{H}$ distances and with $U_{\text {iso }}(\mathrm{H})$ equal to $\left.1.2 U_{\text {eq }}(\mathrm{C})\right)$; for $\mathbf{I V}$ the $\mathrm{H}$-atoms were refined independently.

Atomic positional and displacement parameters for I-IV are given in Tables 2-5. The figures were prepared using DIAMOND 3.1 software [13]. 


\section{Results and discussion}

The $\pi$-complex $\left[\left\{\mathrm{C}_{3} \mathrm{H}_{5} \mathrm{NH}\left(\mathrm{H}^{+}\right) \mathrm{C}_{2} \mathrm{H}_{4} \mathrm{CN}\right\} \mathrm{Cu}_{2} \mathrm{Br}_{3}\right]$ (I) is isostructural to the previously studied copper(I) chloride complex $\left[\left\{\mathrm{C}_{3} \mathrm{H}_{5} \mathrm{NH}\left(\mathrm{H}^{+}\right) \mathrm{C}_{2} \mathrm{H}_{4} \mathrm{CN}\right\} \mathrm{Cu}_{2} \mathrm{Cl}_{3}\right]$ [6]. In the crystal structure of $\mathbf{I}$ the $L 1\left(\mathrm{H}^{+}\right)$cation is coordinated by two copper(I) atoms through the $\mathrm{C}=\mathrm{C}$ bond of an allyl group and an $\mathrm{N}$ atom of a nitrile group. As a result of the bridging function of the organic cations (they are coordinated by metal atoms from parallel inorganic chains) the structure consists of 2D coordination layers (Fig. 1). Two crystallographically independent $\mathrm{Cu}(\mathrm{I})$ atoms possess trigonal-pyramidal coordination environment: $\mathrm{Cu}(1)$ is surrounded by a $\mathrm{C}=\mathrm{C}$-bond and three $\mathrm{Br}$ atoms, $\mathrm{Cu}(2)$ by one $\mathrm{N}$ atom and three halogen atoms. In both cases the apical position of the metal coordination polyhedron is occupied by a halogen atom (Table 6). The infinite metal halide chain $\left(\mathrm{Cu}_{2} \mathrm{Br}_{3}\right)_{n}^{n-}$ of the compound is similar to those in the structure of the related complex $\left[\mathrm{H}^{+}\right.$(diallylamin $\left.) \mathrm{Cu}_{2} \mathrm{Br}_{3}\right]$ [14]. Bonds [15] (N)H...Br and (C)H...Br, formed by $\mathrm{H}$ atoms of the amino-group of $L 1\left(\mathrm{H}^{+}\right)$, connect the polymeric coordination layers mentioned above into a 3D-framework (Table 7).

In the structure of II, the organic cation $\mathrm{L2}_{2}\left(\mathrm{H}^{+}\right)$, which possesses two allyl groups, does

Table 2 Fractional atomic coordinates and isotropic or equivalent isotropic displacement parameters $\left(\AA^{2}\right)$ for $\mathbf{I}$.

\begin{tabular}{l|c|l|l|l|l|l|l|l|l|l}
\hline Atom & $x$ & $y$ & $z$ & $U_{\mathrm{iso}} / U_{\mathrm{eq}}{ }^{\mathrm{a}}$ & Atom & $x$ & $y$ & $U^{2}$ & $U_{\mathrm{iso}} / U_{\mathrm{eq}}{ }^{\mathrm{a}}$ \\
\hline $\mathrm{Cu}(1)$ & $0.77442(12)$ & $0.26878(10)$ & $0.85467(7)$ & $0.0363(2)$ & $\mathrm{H}(1 \mathrm{~N})$ & -0.1206 & 0.0644 & 0.2407 & 0.037 \\
$\mathrm{Cu}(2)$ & $0.40281(13)$ & $0.25313(10)$ & $0.67075(7)$ & $0.0416(2)$ & $\mathrm{H}(2 \mathrm{~N})$ & 0.0706 & 0.1653 & 0.2709 & 0.037 \\
$\mathrm{Br}(1)$ & $0.65575(10)$ & $0.49036(8)$ & $0.71833(5)$ & $0.03659(19)$ & $\mathrm{H}(2 \mathrm{~A})$ & -0.2555 & 0.3388 & 0.4702 & 0.041 \\
$\mathrm{Br}(2)$ & $1.14215(10)$ & $0.24439(8)$ & $0.83787(5)$ & $0.03569(19)$ & $\mathrm{H}(2 \mathrm{~B})$ & -0.2529 & 0.1446 & 0.4387 & 0.041 \\
$\mathrm{Br}(3)$ & $0.60876(10)$ & $0.00760(8)$ & $0.72155(6)$ & $0.0398(2)$ & $\mathrm{H}(3 \mathrm{~A})$ & -0.3110 & 0.2974 & 0.2656 & 0.037 \\
$\mathrm{~N}(1)$ & $0.1767(11)$ & $0.2456(8)$ & $0.5443(5)$ & $0.0489(15)$ & $\mathrm{H}(3 \mathrm{~B})$ & -0.1058 & 0.4092 & 0.2948 & 0.037 \\
$\mathrm{~N}(2)$ & $-0.0570(8)$ & $0.1686(6)$ & $0.2356(4)$ & $0.0310(11)$ & $\mathrm{H}(4 \mathrm{~A})$ & 0.0115 & 0.3278 & 0.0997 & 0.039 \\
$\mathrm{C}(1)$ & $0.0180(11)$ & $0.2494(8)$ & $0.4971(5)$ & $0.0377(16)$ & $\mathrm{H}(4 \mathrm{~B})$ & 0.0513 & 0.1376 & 0.0714 & 0.039 \\
$\mathrm{C}(2)$ & $-0.1787(9)$ & $0.2545(9)$ & $0.4324(5)$ & $0.0341(14)$ & $\mathrm{H}(51)$ & -0.2868 & 0.0820 & 0.0069 & 0.041 \\
$\mathrm{C}(3)$ & $-0.1720(9)$ & $0.2962(7)$ & $0.3020(5)$ & $0.0305(13)$ & $\mathrm{H}(6 \mathrm{~A})$ & -0.3216 & 0.4296 & 0.0420 & 0.044 \\
$\mathrm{C}(4)$ & $-0.0433(9)$ & $0.2109(8)$ & $0.1056(5)$ & $0.0325(14)$ & $\mathrm{H}(6 \mathrm{~B})$ & -0.4886 & 0.3009 & -0.0310 \\
$\mathrm{C}(5)$ & $-0.2418(10)$ & $0.1900(8)$ & $0.0365(5)$ & $0.0339(14)$ & & & & 0.044 \\
$\mathrm{C}(6)$ & $-0.3634(10)$ & $0.3204(8)$ & $0.0134(5)$ & $0.0366(15)$ & & & & \\
\hline
\end{tabular}

${ }^{a}$ For non-hydrogen atoms $U_{\text {eq }}$ is defined as one-third of the trace of the orthogonalized $U_{i j}$ tensor $U_{\text {eq }}=1 / 3 \sum_{i} \sum_{j} U_{i j} a_{i}^{*} a_{j}^{*}\left(\vec{a}_{i} \vec{a}_{j}\right)$, for hydrogen $U_{\text {iso }}$ was set equal to $1.2 U_{\text {eq }}(\mathrm{C})$

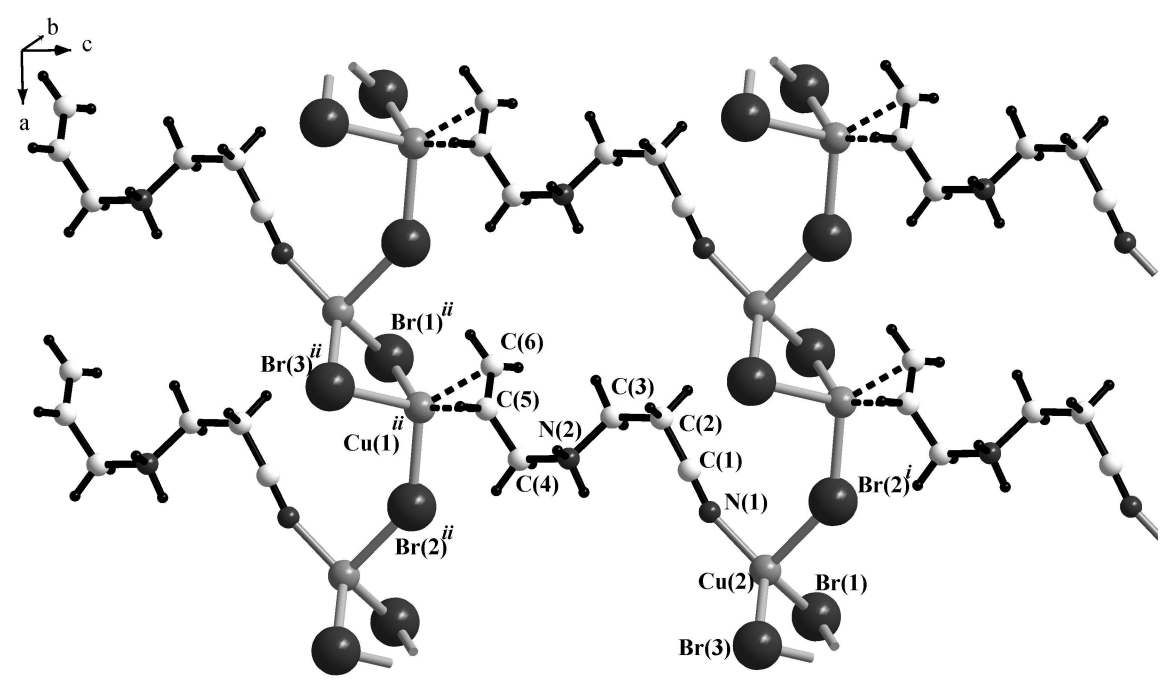

Fig. 1 Projection of the structure of $\left[\left\{\mathrm{C}_{3} \mathrm{H}_{5} \mathrm{NH}\left(\mathrm{H}^{+}\right) \mathrm{C}_{2} \mathrm{H}_{4} \mathrm{CN}\right\} \mathrm{Cu}_{2} \mathrm{Br}_{3}\right](\mathbf{I})$. Symmetry codes: $(i)-1+x, y, z,(i i)-1+x, y,-1+z$. 
not demonstrate its multiple coordination possibilities (bridging or chelate behaviour is not present). It is coordinated to copper(I) by the $\mathrm{C}=\mathrm{C}$ bond of only one allyl group. The nitrile group and the $\mathrm{C}=\mathrm{C}$ bond from the other allyl radical do not take part in the metal coordination (Fig. 2). The copper atom possesses trigonal pyramidal coordination, formed by one $\mathrm{C}=\mathrm{C}$ bond and three halogen atoms. The distance $\mathrm{Cu}-$ $(\mathrm{C}=\mathrm{C})$ is $1.945(5) \AA, d\left(\mathrm{Cu}-\mathrm{X}_{\mathrm{ap}}\right)=2.8505(10) \AA$; the distances $\mathrm{Cu}-\mathrm{X}$ are 2.2549(9) and 2.2853(10) $\AA$, the angles $\mathrm{X}-\mathrm{Cu}-\mathrm{X} / \mathrm{X}-\mathrm{Cu}-m$ are in the range $92-101^{\circ}$ (Table 8). Due to the bridging role of the halogen atoms, copper(I) halide units are connected into polymeric $\left(\mathrm{CuX}_{2}\right)_{n}^{n-}$ chains, which are orientated along the $b$-axis. $\pi$-coordinated monodentate cations $L 2\left(\mathrm{H}^{+}\right)$are attached to the anionic copper(I) halide fragments, forming $1 \mathrm{D}$ coordination polymers. A branched system of H-bonds [16,17] connects the separate "chains" into a framework (Fig. 3).

In the case of compound III, the organic ligand

Table 3 Fractional atomic coordinates and isotropic or equivalent isotropic displacement parameters $\left(\AA^{2}\right)$ for $\mathbf{I I}$.

\begin{tabular}{|c|c|c|c|c|}
\hline Atom & $x$ & $y$ & $z$ & $U_{\text {iso }} / U_{\text {eq }}{ }^{\mathrm{a}}$ \\
\hline $\mathrm{Cu}$ & $0.52101(4)$ & $0.05441(7)$ & $0.36225(3)$ & $0.02458(18)$ \\
\hline$X(1)^{b}$ & $0.71705(7)$ & $0.07566(13)$ & $0.40437(7)$ & $0.0295(3)$ \\
\hline$X(2)^{b}$ & $0.47908(7)$ & $-0.24784(13)$ & $0.30748(6)$ & $0.0249(3)$ \\
\hline $\mathrm{N}(1)$ & $0.1219(4)$ & $0.0584(6)$ & $-0.0842(3)$ & $0.0432(10)$ \\
\hline $\mathrm{N}(2)$ & $0.2429(3)$ & $-0.0285(5)$ & $0.2454(2)$ & $0.0207(6)$ \\
\hline $\mathrm{C}(1)$ & $0.1785(4)$ & $0.0620(6)$ & $-0.0123(3)$ & $0.0287(9)$ \\
\hline $\mathrm{C}(2)$ & $0.2490(3)$ & $0.0640(6)$ & $0.0816(3)$ & $0.0246(8)$ \\
\hline $\mathrm{C}(3)$ & $0.1720(3)$ & $0.0005(6)$ & $0.1494(3)$ & $0.0232(7)$ \\
\hline $\mathrm{C}(4)$ & $0.2723(3)$ & $0.1597(6)$ & $0.2944(3)$ & $0.0212(7)$ \\
\hline$C(5)$ & $0.3588(3)$ & $0.1424(6)$ & $0.3840(2)$ & $0.0225(7)$ \\
\hline$C(6)$ & $0.4403(3)$ & $0.2789(6)$ & $0.4142(3)$ & $0.0266(8)$ \\
\hline$C(7)$ & $0.1791(3)$ & $-0.1650(6)$ & $0.3002(3)$ & $0.0255(8)$ \\
\hline $\mathrm{C}(8)$ & $0.0598(3)$ & $-0.0950(6)$ & $0.3063(3)$ & $0.0293(9)$ \\
\hline $\mathrm{C}(9)$ & $0.0306(4)$ & $-0.0496(8)$ & $0.3855(3)$ & $0.0392(11)$ \\
\hline $\mathrm{H}(1 \mathrm{~N})$ & $0.311(4)$ & $-0.093(7)$ & $0.242(3)$ & $0.030(12)$ \\
\hline $\mathrm{H}(2 \mathrm{~A})$ & $0.309(4)$ & $-0.028(7)$ & $0.079(3)$ & $0.036(13)$ \\
\hline $\mathrm{H}(2 \mathrm{~B})$ & $0.273(4)$ & $0.196(8)$ & $0.090(3)$ & $0.038(13)$ \\
\hline $\mathrm{H}(3 \mathrm{~A})$ & $0.110(4)$ & $0.090(6)$ & $0.153(3)$ & $0.025(11)$ \\
\hline $\mathrm{H}(3 \mathrm{~B})$ & $0.139(4)$ & $-0.117(7)$ & $0.130(3)$ & $0.020(10)$ \\
\hline $\mathrm{H}(4 \mathrm{~A})$ & $0.299(4)$ & $0.235(7)$ & $0.257(3)$ & $0.023(11)$ \\
\hline $\mathrm{H}(4 \mathrm{~B})$ & $0.199(4)$ & $0.206(6)$ & $0.305(3)$ & $0.021(10)$ \\
\hline $\mathrm{H}(51)$ & $0.345(4)$ & $0.058(7)$ & $0.430(4)$ & $0.038(13)$ \\
\hline $\mathrm{H}(6 \mathrm{~A})$ & $0.446(3)$ & $0.387(6)$ & $0.385(3)$ & $0.014(9)$ \\
\hline $\mathrm{H}(6 \mathrm{~B})$ & $0.480(5)$ & $0.285(8)$ & $0.476(4)$ & $0.053(16)$ \\
\hline $\mathrm{H}(7 \mathrm{~A})$ & $0.179(4)$ & $-0.284(7)$ & $0.267(3)$ & $0.023(11)$ \\
\hline $\mathrm{H}(7 \mathrm{~B})$ & $0.228(4)$ & $-0.184(7)$ & $0.360(3)$ & $0.028(11)$ \\
\hline $\mathrm{H}(81)$ & $0.007(5)$ & $-0.094(7)$ & $0.252(4)$ & $0.035(12)$ \\
\hline $\mathrm{H}(9 \mathrm{~A})$ & $-0.050(5)$ & $-0.013(9)$ & $0.391(4)$ & $0.057(16)$ \\
\hline $\mathrm{H}(9 \mathrm{~B})$ & $0.087(5)$ & $-0.058(8)$ & $0.446(4)$ & $0.055(17)$ \\
\hline
\end{tabular}

\footnotetext{
$U_{\mathrm{eq}}=1 / 3 \sum_{i} \sum_{j} U_{i j} a_{i}^{*} a_{j}^{*}\left(\vec{a}_{i} \vec{a}_{j}\right)$, for hydrogen $U_{\text {iso }}$ was set equal to $1.2 U_{\mathrm{eq}}(\mathrm{C})$.

${ }^{\mathrm{b}} \mathrm{X}(1) \equiv(0.959(4) \mathrm{Cl}+0.041(4) \mathrm{Br}) ; \mathrm{X}(2) \equiv(0.979(4) \mathrm{Cl}+0.021(4) \mathrm{Br})$.
}

${ }^{\mathrm{a}}$ For non-hydrogen atoms $U_{\mathrm{eq}}$ is defined as one-third of the trace of the orthogonalized $U_{i j}$ tensor 
Table 4 Fractional atomic coordinates and isotropic or equivalent isotropic displacement parameters $\left(\AA^{2}\right)$ for III.

\begin{tabular}{|c|c|c|c|c|c|}
\hline Atom & $x$ & $y$ & $z$ & $U_{\mathrm{iso}} / U_{\mathrm{eq}}{ }^{\mathrm{a}}$ & Occ. $(<1)$ \\
\hline $\mathrm{Cu}$ & $0.24472(4)$ & $0.34383(4)$ & $0.162448(17)$ & $0.03562(9)$ & \\
\hline $\mathrm{Cl}(1)$ & 0 & $0.34116(9)$ & $1 / 4$ & $0.02612(13)$ & \\
\hline $\mathrm{Cl}(2)$ & $0.29078(7)$ & $0.05214(7)$ & $0.13757(3)$ & $0.03635(13)$ & \\
\hline $\mathrm{Cl}(31)$ & $1 / 2$ & $0.478(3)$ & $1 / 4$ & $0.032(2)$ & $0.45(5)$ \\
\hline $\mathrm{Cl}(3)$ & $0.4868(8)$ & $0.4402(10)$ & $0.2698(9)$ & $0.0202(12)$ & $0.27(3)$ \\
\hline $\mathrm{O}(1)$ & $0.3907(3)$ & $1.2914(3)$ & $-0.08896(15)$ & $0.0361(7)$ & $0.661(3)$ \\
\hline $\mathrm{O}(11)$ & $0.4111(6)$ & $1.2922(6)$ & $-0.1273(3)$ & $0.0261(10)$ & $0.339(3)$ \\
\hline $\mathrm{N}(1)$ & $0.2254(7)$ & $0.5114(7)$ & $0.0612(3)$ & $0.0306(10)$ & $0.661(3)$ \\
\hline $\mathrm{N}(11)$ & $0.2019(14)$ & $0.5033(11)$ & $0.0861(5)$ & $0.0256(16)$ & $0.339(3)$ \\
\hline $\mathrm{N}(2)$ & $0.2696(5)$ & $0.9585(4)$ & $-0.1535(2)$ & $0.0233(7)$ & $0.661(3)$ \\
\hline $\mathrm{N}(21)$ & $0.3093(10)$ & $0.9259(7)$ & $-0.1307(4)$ & $0.0231(15)$ & $0.339(3)$ \\
\hline $\mathrm{C}(1)$ & $0.2611(4)$ & $0.5950(4)$ & $0.0056(2)$ & $0.0284(7)$ & $0.661(3)$ \\
\hline $\mathrm{C}(11)$ & $0.1830(7)$ & $0.6083(7)$ & $0.0349(3)$ & $0.0202(11)$ & $0.339(3)$ \\
\hline $\mathrm{C}(2)$ & $0.3144(5)$ & $0.6941(4)$ & $-0.0670(2)$ & $0.0306(7)$ & $0.661(3)$ \\
\hline $\mathrm{C}(21)$ & $0.1578(6)$ & $0.7415(7)$ & $-0.0317(3)$ & $0.0227(11)$ & $0.339(3)$ \\
\hline $\mathrm{C}(3)$ & $0.2209(4)$ & $0.8711(3)$ & $-0.07497(17)$ & $0.0242(6)$ & $0.661(3)$ \\
\hline $\mathrm{C}(31)$ & $0.3376(6)$ & $0.8020(7)$ & $-0.0589(3)$ & $0.0215(11)$ & $0.339(3)$ \\
\hline $\mathrm{C}(4)$ & $0.1672(4)$ & $1.1232(4)$ & $-0.1740(2)$ & $0.0301(7)$ & $0.661(3)$ \\
\hline $\mathrm{C}(41)$ & $0.1879(7)$ & $1.0758(6)$ & $-0.1150(3)$ & $0.0241(12)$ & $0.339(3)$ \\
\hline $\mathrm{C}(5)$ & $0.2037(3)$ & $1.2597(3)$ & $-0.10632(15)$ & $0.0356(8)$ & $0.661(3)$ \\
\hline $\mathrm{C}(51)$ & $0.2385(6)$ & $1.2365(6)$ & $-0.1621(3)$ & $0.0261(12)$ & $0.339(3)$ \\
\hline $\mathrm{H}(1 \mathrm{O})$ & 0.4318 & 1.3326 & -0.1323 & 0.054 & $0.661(3)$ \\
\hline $\mathrm{H}(110)$ & 0.4547 & 1.3618 & -0.1609 & 0.039 & $0.339(3)$ \\
\hline $\mathrm{H}(2 \mathrm{~A})$ & 0.2836 & 0.6260 & -0.1194 & 0.037 & $0.661(3)$ \\
\hline $\mathrm{H}(21 \mathrm{~A})$ & 0.0927 & 0.8429 & -0.0105 & 0.027 & $0.339(3)$ \\
\hline $\mathrm{H}(2 \mathrm{~B})$ & 0.4466 & 0.7119 & -0.0605 & 0.037 & $0.661(3)$ \\
\hline $\mathrm{H}(21 \mathrm{~B})$ & 0.0835 & 0.6925 & -0.0811 & 0.027 & $0.339(3)$ \\
\hline $\mathrm{H}(3 \mathrm{~A})$ & 0.0887 & 0.8552 & -0.0776 & 0.029 & $0.661(3)$ \\
\hline $\mathrm{H}(31 \mathrm{~A})$ & 0.4082 & 0.8598 & -0.0107 & 0.026 & $0.339(3)$ \\
\hline $\mathrm{H}(3 \mathrm{~B})$ & 0.2594 & 0.9444 & -0.0251 & 0.029 & $0.661(3)$ \\
\hline $\mathrm{H}(31 \mathrm{~B})$ & 0.4070 & 0.6996 & -0.0760 & 0.026 & $0.339(3)$ \\
\hline $\mathrm{H}(4 \mathrm{~A})$ & 0.0366 & 1.0968 & -0.1808 & 0.036 & $0.661(3)$ \\
\hline $\mathrm{H}(41 \mathrm{~A})$ & 0.1962 & 1.1013 & -0.0536 & 0.029 & $0.339(3)$ \\
\hline $\mathrm{H}(4 \mathrm{~B})$ & 0.2011 & 1.1701 & -0.2285 & 0.036 & $0.661(3)$ \\
\hline $\mathrm{H}(41 \mathrm{~B})$ & 0.0616 & 1.0434 & -0.1335 & 0.029 & $0.339(3)$ \\
\hline $\mathrm{H}(5 \mathrm{~A})$ & 0.1423 & 1.3700 & -0.1249 & 0.043 & $0.661(3)$ \\
\hline $\mathrm{H}(51 \mathrm{~A})$ & 0.2411 & 1.2094 & -0.2230 & 0.031 & $0.339(3)$ \\
\hline $\mathrm{H}(5 \mathrm{~B})$ & 0.1536 & 1.2202 & -0.0539 & 0.043 & $0.661(3)$ \\
\hline $\mathrm{H}(51 \mathrm{~B})$ & 0.1488 & 1.3303 & -0.1565 & 0.031 & $0.339(3)$ \\
\hline $\mathrm{H}(1 \mathrm{~N})$ & 0.2488 & 0.8820 & -0.1981 & 0.028 & $0.661(3)$ \\
\hline $\mathrm{H}(11 \mathrm{~N})$ & 0.2611 & 0.8659 & -0.1778 & 0.028 & $0.339(3)$ \\
\hline $\mathrm{H}(2 \mathrm{~N})$ & 0.3908 & 0.9837 & -0.1475 & 0.028 & $0.661(3)$ \\
\hline $\mathrm{H}(21 \mathrm{~N})$ & 0.4193 & 0.9695 & -0.1424 & 0.028 & $0.339(3)$ \\
\hline
\end{tabular}

${ }^{a}$ For non-hydrogen atoms $U_{\text {eq }}$ is defined as one-third of the trace of the orthogonalized $U_{i j}$ tensor $U_{\mathrm{eq}}=1 / 3 \sum_{i} \sum_{j} U_{i j} a_{i}^{*} a_{j}^{*}\left(\vec{a}_{i} \vec{a}_{j}\right)$, for hydrogen $U_{\text {iso }}$ was set equal to $1.2 U_{\mathrm{eq}}(\mathrm{C})$. 
Table 5 Fractional atomic coordinates and isotropic or equivalent isotropic displacement parameters $\left(\AA^{2}\right)$ for IV.

\begin{tabular}{l|c|l|l|l||l|l|l|l|l|l}
\hline Atom & $x$ & $y$ & $z$ & $U_{\text {iso }} U_{\mathrm{eq}}{ }^{\mathrm{a}}$ & Atom & $x$ & $y$ & $z$ & $U_{\text {iso }}$ \\
\hline $\mathrm{Cu}$ & $0.52517(18)$ & $0.43239(3)$ & $0.364852(12)$ & $0.01384(6)$ & $\mathrm{H}(1 \mathrm{~N})$ & 0.3039 & 0.5749 & 0.2568 & 0.017 \\
$\mathrm{Cl}(1)$ & $0.47769(3)$ & $0.72819(5)$ & $0.30685(2)$ & $0.01212(8)$ & $\mathrm{H}(1 \mathrm{O})$ & -0.0974 & 0.4833 & 0.3304 & 0.036 \\
$\mathrm{Cl}(2)$ & $0.73163(3)$ & $0.41169(5)$ & $0.40078(2)$ & $0.01387(8)$ & $\mathrm{H}(2 \mathrm{~A})$ & 0.3081 & 0.5117 & 0.1150 & 0.021 \\
$\mathrm{~N}(1)$ & $0.11676(6)$ & $0.45738(10)$ & $-0.05489(4)$ & $0.0270(3)$ & $\mathrm{H}(2 \mathrm{~B})$ & 0.2607 & 0.2967 & 0.1213 & 0.021 \\
$\mathrm{~N}(2)$ & $0.22811(6)$ & $0.51147(10)$ & $0.27420(4)$ & $0.0136(2)$ & $\mathrm{H}(3 \mathrm{~A})$ & 0.1123 & 0.6147 & 0.1640 & 0.018 \\
$\mathrm{O}(1)$ & $-0.03711(6)$ & $0.52958(10)$ & $0.30821(4)$ & $0.0243(3)$ & $\mathrm{H}(3 \mathrm{~B})$ & 0.0872 & 0.3952 & 0.1859 & 0.018 \\
$\mathrm{C}(1)$ & $0.16764(6)$ & $0.44684(10)$ & $0.01764(4)$ & $0.0181(3)$ & $\mathrm{H}(4 \mathrm{~A})$ & 0.1929 & 0.2507 & 0.3311 & 0.017 \\
$\mathrm{C}(2)$ & $0.23406(6)$ & $0.43026(10)$ & $0.10990(4)$ & $0.0171(3)$ & $\mathrm{H}(4 \mathrm{~B})$ & 0.3015 & 0.2412 & 0.2682 & 0.017 \\
$\mathrm{C}(3)$ & $0.15211(15)$ & $0.4915(2)$ & $0.18215(10)$ & $0.0153(3)$ & $\mathrm{H}(51)$ & 0.3549 & 0.4424 & 0.4382 & 0.016 \\
$\mathrm{C}(4)$ & $0.26624(15)$ & $0.3183(2)$ & $0.31495(10)$ & $0.0138(3)$ & $\mathrm{H}(6 \mathrm{~A})$ & 0.4534 & 0.0927 & 0.3844 & 0.019 \\
$\mathrm{C}(5)$ & $0.35867(14)$ & $0.3348(2)$ & $0.39929(10)$ & $0.0130(3)$ & $\mathrm{H}(6 \mathrm{~B})$ & 0.5046 & 0.2175 & 0.4763 & 0.019 \\
$\mathrm{C}(6)$ & $0.44797(15)$ & $0.2014(2)$ & $0.42238(10)$ & $0.0158(3)$ & $\mathrm{H}(7 \mathrm{~A})$ & 0.2322 & 0.6759 & 0.3905 & 0.020 \\
$\mathrm{C}(7)$ & $0.16907(11)$ & $0.63981(16)$ & $0.33922(8)$ & $0.0165(3)$ & $\mathrm{H}(7 \mathrm{~B})$ & 0.1420 & 0.7581 & 0.3059 & 0.020 \\
$\mathrm{C}(8)$ & $0.06107(11)$ & $0.55665(16)$ & $0.37940(8)$ & $0.0184(3)$ & $\mathrm{H}(8 \mathrm{~A})$ & 0.0843 & 0.4332 & 0.4093 & 0.022 \\
& & & & & & & & &
\end{tabular}

${ }^{\mathrm{a}}$ For non-hydrogen atoms $U_{\mathrm{eq}}$ is defined as one-third of the trace of the orthogonalized $U_{i j}$ tensor $U_{\text {eq }}=1 / 3 \sum_{i} \sum_{j} U_{i j} a_{i}^{*} a_{j}^{*}\left(\vec{a}_{i} \vec{a}_{j}\right)$, for hydrogen $U_{\text {iso }}$ was set equal to $1.2 U_{\mathrm{eq}}(\mathrm{C})$.

Table 6 Selected bond lengths and angles in the structures of $\mathbf{I}$.

\begin{tabular}{|c|c|c|c|c|c|c|c|}
\hline Bond & $d, \AA$ & Angle & $\omega$, deg & Bond & $d, \AA$ & Angle & $\omega, \operatorname{deg}$ \\
\hline $\mathrm{Cu}(1)-m^{a}$ & $2.019(8)$ & $\mathrm{Cu}(2)-\mathrm{Br}(1)-\mathrm{Cu}(1)$ & $77.46(3)$ & $\operatorname{Br}(3)-\mathrm{Cu}(2)$ & $2.4735(10)$ & $\mathrm{N}(1)-\mathrm{Cu}(2)-\mathrm{Br}(1)$ & $126.61(19)$ \\
\hline $\mathrm{Br}(2)-\mathrm{Cu}(2)^{i l}$ & $2.6327(10)$ & $\mathrm{Cu}(1)-\mathrm{Br}(2)-\mathrm{Cu}(2)^{i}$ & $138.55(4)$ & $\mathrm{Cu}(2)-\mathrm{N}(1)$ & $1.970(7)$ & $\mathrm{N}(1)-\mathrm{Cu}(2)-\mathrm{Br}(3)$ & $123.58(18)$ \\
\hline $\mathrm{Cu}(1)-\mathrm{C}(6)^{i i}$ & $2.107(6)$ & $\mathrm{Cu}(2)-\mathrm{Br}(3)-\mathrm{Cu}(1)$ & $73.39(3)$ & $\mathrm{N}(1)-\mathrm{C}(1)$ & $1.133(9)$ & $\mathrm{Br}(1)-\mathrm{Cu}(2)-\mathrm{Br}(3)$ & $100.94(4)$ \\
\hline $\mathrm{Cu}(1)-\mathrm{C}(5)^{i i}$ & $2.150(6)$ & $\mathrm{C}(6)^{\mathrm{ii}}-\mathrm{Cu}(1)-\mathrm{C}(5)^{i i}$ & $37.1(2)$ & $\mathrm{N}(2)-\mathrm{C}(4)$ & $1.510(6)$ & $\mathrm{N}(1)-\mathrm{Cu}(2)-\mathrm{Br}(2)^{i i i}$ & $91.33(17)$ \\
\hline $\operatorname{Br}(1)-\mathrm{Cu}(1)$ & $2.4583(10)$ & $\mathrm{C}(6)^{i i}-\mathrm{Cu}(1)-\mathrm{Br}(2)$ & $125.56(18)$ & $\mathrm{C}(4)-\mathrm{C}(5)$ & $1.461(8)$ & $\operatorname{Br}(1)-\mathrm{Cu}(2)-\mathrm{Br}(2)^{i i i}$ & $107.71(4)$ \\
\hline $\mathrm{Br}(2)-\mathrm{Cu}(1)$ & $2.4508(11)$ & $\mathrm{C}(5)^{i i}-\mathrm{Cu}(1)-\mathrm{Br}(2)$ & $98.80(17)$ & $C(5)=C(6)$ & $1.354(8)$ & $\mathrm{Br}(3)-\mathrm{Cu}(2)-\mathrm{Br}(2)^{i i i}$ & $102.25(4)$ \\
\hline $\mathrm{Br}(3)-\mathrm{Cu}(1)$ & $2.6500(10)$ & $\mathrm{C}(6)^{i i}-\mathrm{Cu}(1)-\mathrm{Br}(1)$ & $103.75(17)$ & $\mathrm{N}(2)-\mathrm{C}(3)$ & $1.516(7)$ & $\mathrm{C}(4)-\mathrm{N}(2)-\mathrm{C}(3)$ & $112.9(4)$ \\
\hline \multirow[t]{6}{*}{$\mathrm{Br}(1)-\mathrm{Cu}(2)$} & $2.4401(11)$ & $\mathrm{C}(5)^{\mathrm{ii}}-\mathrm{Cu}(1)-\mathrm{Br}(1)$ & $140.69(16)$ & $C(2)-C(3)$ & $1.510(7)$ & $\mathrm{N}(1)-\mathrm{C}(1)-\mathrm{C}(2)$ & $177.5(7)$ \\
\hline & & $\operatorname{Br}(2)-\mathrm{Cu}(1)-\mathrm{Br}(1)$ & $108.62(4)$ & $C(1)-C(2)$ & $1.433(9)$ & $\mathrm{C}(1)-\mathrm{C}(2)-\mathrm{C}(3)$ & $114.8(5)$ \\
\hline & & $\mathrm{C}(6)^{i i}-\mathrm{Cu}(1)-\mathrm{Br}(3)$ & $116.79(19)$ & & & $\mathrm{C}(5)-\mathrm{C}(4)-\mathrm{N}(2)$ & $112.7(5)$ \\
\hline & & $\mathrm{C}(5)^{i i}-\mathrm{Cu}(1)-\mathrm{Br}(3)$ & $105.54(16)$ & & & $C(6)-C(5)-C(4)$ & $123.1(6)$ \\
\hline & & $\mathrm{Br}(2)-\mathrm{Cu}(1)-\mathrm{Br}(3)$ & $102.26(3)$ & & & $\mathrm{N}(2)-\mathrm{C}(3)-\mathrm{C}(2)$ & $112.0(5)$ \\
\hline & & $\operatorname{Br}(1)-\mathrm{Cu}(1)-\mathrm{Br}(3)$ & $95.72(3)$ & & & & \\
\hline
\end{tabular}

${ }^{\mathrm{a}} m$ - middle point of the $\mathrm{C}(5)=\mathrm{C}(6)$ bond.

${ }^{\mathrm{b}}$ Symmetry codes: (i) $x+1, y, z$; (ii) $x+1, y, z+1$; (iii) $x-1, y, z$.

$L 3\left(H^{+}\right)$(being disordered) exhibits a monodentate behaviour. It is coordinated to the $\mathrm{Cu}(\mathrm{I})$ atom by only the $\mathrm{N}$ atom of a $\mathrm{CN}$ group, whereas the hydroxyl group stays uncoordinated (Fig. 4). The crystal structure contains infinite inorganic chains $\left(\mathrm{CuCl}_{2}\right)_{n}^{n-}$. In contrast to the structure of $\mathbf{I}$, it possesses only one independent copper(I) atom, which has a distorted tetrahedral coordination with one nitrogen and three chlorine atoms. The distance $\mathrm{Cu}-\mathrm{N}$ is $2.049(4) \AA, \quad d(\mathrm{Cu}-\mathrm{Cl})=$ 2.2952(8), 2.3949(8) and 2.473(5) $\AA$, the angles $\mathrm{Cl}-\mathrm{Cu}-\mathrm{Cl}$ are in the range $103.1-114.3(4)^{\circ}, \mathrm{Cl}-\mathrm{Cu}-\mathrm{N}$ $101.3-125.8^{\circ}$ (Table 9). The cations $L 3\left(H^{+}\right)$are attached to an anionic chain on one side of the plane formed by $\mathrm{Cu}$ atoms. One-dimensional coordination polymers are combined into a framework by weak $\mathrm{H}$ bonds. 
Modification of 3-(\{2-hydroxyethyl $\}$ amino $)$ propanenitrile by adding an allyl group has given one more coordination centre, but in the structure of IV 3-(\{2-hydroxyethyl $\}$ allylamino)propanenitrile also behaves as a monodentate ligand, although in this case it is attached to $\mathrm{Cu}(\mathrm{I})$ by the $\mathrm{C}=\mathrm{C}$ bond of an allyl group (not the $\mathrm{N}$ atom of a $\mathrm{CN}$ group, Fig. 5).The metal atom has trigonal pyramidal coordination formed by one $\mathrm{C}=\mathrm{C}$ bond and three chlorine atoms. The distance $\mathrm{Cu}-(\mathrm{C}=\mathrm{C})$ is $1.9505(5) \AA, d\left(\mathrm{Cu}-\mathrm{Cl}_{\mathrm{ap}}\right)=$ 2.892(5) $\AA$; the other $\mathrm{Cu}-\mathrm{Cl}$ contacts are 2.2786(7) and $2.2715(8) \AA$, the angles $\mathrm{Cl}-\mathrm{Cu}-\mathrm{Cl} / \mathrm{Cl}-\mathrm{Cu}-\mathrm{m}$ are in the range $94.1-126.3^{\circ}$ (Table 10). In spite of the differences in the coordination behaviour of $\mathrm{L2}_{2}\left(\mathrm{H}^{+}\right)$, $L 3\left(H^{+}\right)$and $L 4\left(H^{+}\right)$, the structure of $\mathbf{I V}$, as well as II

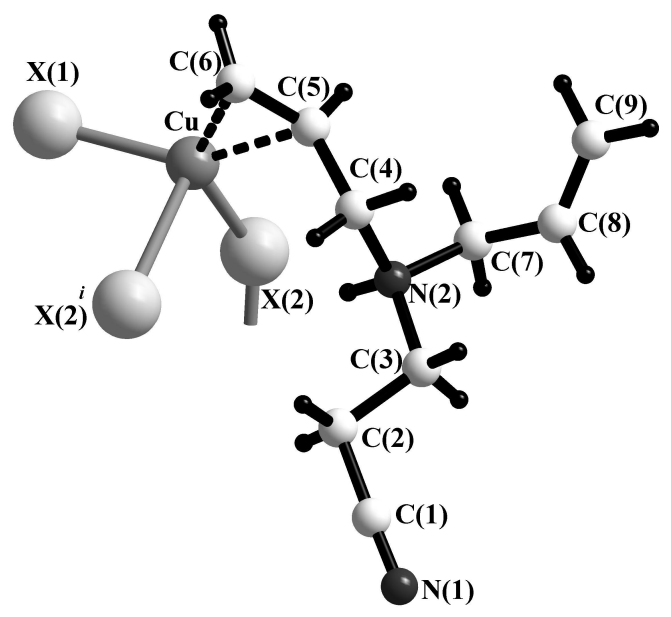

Fig. 2 Fragment of the structure of $\left[\left\{\left(\mathrm{C}_{3} \mathrm{H}_{5}\right)_{2} \mathrm{~N}\left(\mathrm{H}^{+}\right) \mathrm{C}_{2} \mathrm{H}_{4} \mathrm{CN}\right\} \mathrm{CuCl}_{1.94} \mathrm{Br}_{0.06}\right]$ (II).

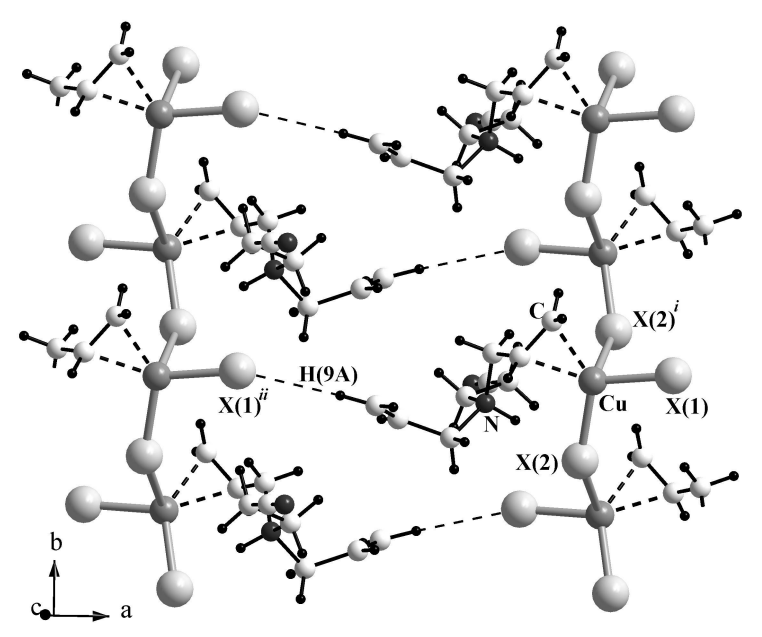

Fig. 3 Projection of the structure of $\left[\left\{\left(\mathrm{C}_{3} \mathrm{H}_{5}\right)_{2} \mathrm{~N}\left(\mathrm{H}^{+}\right) \mathrm{C}_{2} \mathrm{H}_{4} \mathrm{CN}\right\} \mathrm{CuCl}_{1.94} \mathrm{Br}_{0.06}\right]$ $\mathrm{X}$ - Hal.

Symmetry codes: (i) $1-x, 1 / 2+y, 1 / 2-z$; (ii) $-1+x, y, z$. and III, contains an infinite inorganic anion $\left(\mathrm{CuCl}_{2}\right)_{n}^{n-}$. The structures of the complexes II and IV are isotopological: one-dimensional structural fragments with branched organic "attachments" are interconnected by hydrogen bonds and weak intermolecular interactions (Fig. 6).

Some similarities and differences can thus be emphasized. In spite of the different coordination behaviour of $L 1\left(H^{+}\right)$and $L 3\left(H^{+}\right)$in the crystal structures of compounds I and III, the nitrile groups of the organic ligands that have only one substituent at the nitrogen atom of the amino-group, are coordinated by copper(I) atoms. In the case of II and IV, where the organic ligands possess two substituents

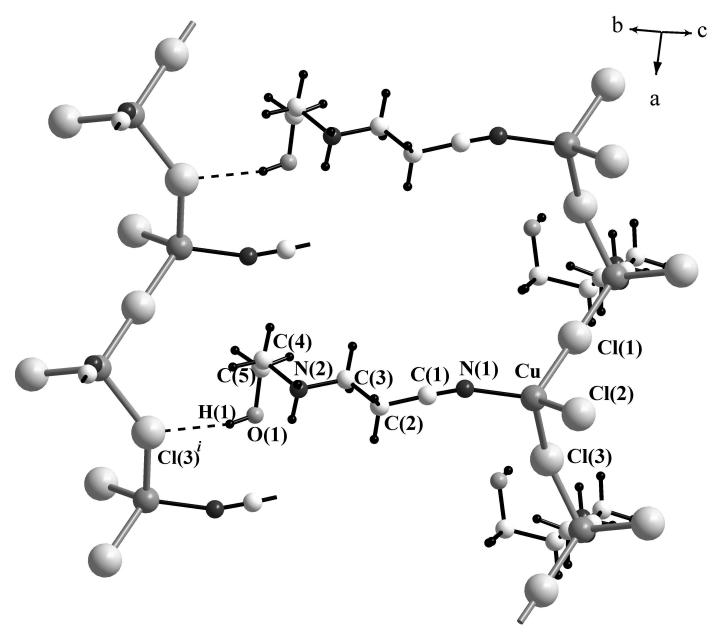

Fig. 4 Fragment of the structure of $\left[\left(\mathrm{HOC}_{2} \mathrm{H}_{5} \mathrm{NH}\left(\mathrm{H}^{+}\right) \mathrm{C}_{2} \mathrm{H}_{4} \mathrm{CN}\right) \mathrm{CuCl}_{2}\right]$ (III). Symmetry code: (i) $x, 2-y,-1 / 2+z$.

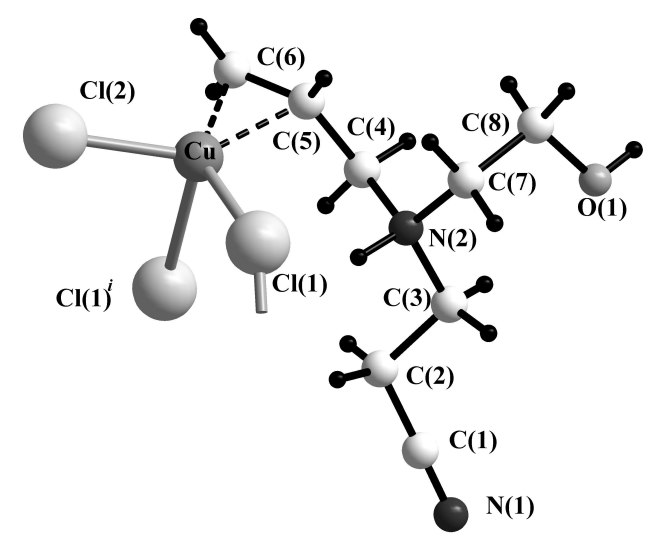

Fig. 5 Fragment of the structure of $\left[\left\{\mathrm{HOC}_{2} \mathrm{H}_{4}\left(\mathrm{C}_{3} \mathrm{H}_{5}\right) \mathrm{N}\left(\mathrm{H}^{+}\right) \mathrm{C}_{2} \mathrm{H}_{4} \mathrm{CN}\right\} \mathrm{CuCl}_{2}\right](\mathbf{I V})$. Symmetry code: (i) $1-x,-1 / 2+y, 1 / 2-z$ 
at the nitrogen atom, the copper(I) atom does not coordinate the $\mathrm{CN}$ group, which is not typical for nitriles. The anomalous inactivity of the $\mathrm{Cu}(\mathrm{I})$ atom to the nitrile group of $\mathrm{N}$-derivatives of propanenitrile disappears in the case of ionic salts of copper(I) [18]. Similarities in the crystal packing of II and IV show that the character of uncoordinated groups at the $\mathrm{N}$ atom has no principal significance in the structure topology, although the presence of the 2-hydroxyethyl substituent leads to the formation of a more complicated system of hydrogen bonds.

Table 7 Geometry of selected H-contacts in the structures of I-IV.

\begin{tabular}{|c|c|c|c|c|c|c|}
\hline \multirow{2}{*}{ No. } & \multirow{2}{*}{ Contact $D-\mathrm{H} . . . A$} & \multicolumn{3}{|c|}{ Bond length, $\AA$} & \multirow{2}{*}{$\begin{array}{c}\text { Angle } D-\mathrm{H} \ldots A \text {, } \\
\text { deg }\end{array}$} & \multirow{2}{*}{$\begin{array}{c}\text { Atom coordinates } \\
\text { of } A\end{array}$} \\
\hline & & $\mathrm{D}-\mathrm{H}$ & H...A & D...A & & \\
\hline \multirow{3}{*}{ I } & $\mathrm{N}(2)-\mathrm{H}(1 \mathrm{~N}) \cdots \mathrm{Br}(2)$ & 0.90 & 2.57 & $3.343(2)$ & 145 & $2-x,-y,-z$ \\
\hline & $\mathrm{N}(2)-\mathrm{H}(2 \mathrm{~N}) \cdots \mathrm{Br}(3)$ & 0.90 & 2.57 & $3.332(3)$ & 143 & $2-x,-y,-z$ \\
\hline & $\mathrm{C}(3)-\mathrm{H}(3 \mathrm{~A}) \cdots \mathrm{Br}(1)$ & 0.97 & 2.91 & $3.674(5)$ & 137 & $1-x, 1-y,-z$ \\
\hline \multirow{3}{*}{ II } & $\mathrm{C}(9)-\mathrm{H}(9 \mathrm{~A}) \cdot \mathrm{X}(1)^{\mathrm{a}}$ & 0.99 & 2.83 & $3.818(5)$ & 178 & $x-1, y, z$ \\
\hline & $\mathrm{C}(4)-\mathrm{H}(4 \mathrm{~B}) \cdots \mathrm{N}(1)$ & 0.95 & 2.59 & $3.367(5)$ & 139 & $x,-y+1 / 2, z+1 / 2$ \\
\hline & $\mathrm{C}(5)-\mathrm{H}(51) \cdots \mathrm{X}(1)^{\mathrm{a}}$ & 0.93 & 2.83 & $3.716(4)$ & 160 & $-x+1,-y,-z+1$ \\
\hline \multirow{3}{*}{ III } & $\mathrm{N}(2)-\mathrm{H}(1 \mathrm{~N}) \cdots \mathrm{Cl}(1)$ & 0.92 & 2.60 & $3.323(4)$ & 136 & $-x,-y+1,-z$ \\
\hline & $\mathrm{N}(2)-\mathrm{H}(2 \mathrm{~N}) \cdots \mathrm{Cl}(2)$ & 0.92 & 2.38 & $3.263(4)$ & 161 & $-x+1,-y+1,-z$ \\
\hline & $\mathrm{C}(3)-\mathrm{H}(3 \mathrm{~B}) \cdots \mathrm{Cl}(2)$ & 0.99 & 2.70 & $3.637(3)$ & 159 & $x, y+1, z$ \\
\hline \multirow{6}{*}{ IV } & $\mathrm{N}(2)-\mathrm{H}(1 \mathrm{~N}) \cdots \mathrm{Cl}(1)$ & 1.01 & 2.24 & $3.123(2)$ & 146 & $x, y, z$ \\
\hline & $\mathrm{O}(1)-\mathrm{H}(1 \mathrm{O}) \cdots \mathrm{Cl}(2)$ & 0.84 & 2.31 & $3.140(3)$ & 170 & $x-1, y, z$ \\
\hline & $\mathrm{C}(4)-\mathrm{H}(4 \mathrm{~A}) \cdots \mathrm{N}(1)$ & 0.99 & 2.44 & $3.297(9)$ & 144 & $x,-y+1 / 2, z+1 / 2$ \\
\hline & $\mathrm{C}(5)-\mathrm{H}(51) \cdots \mathrm{Cl}(2)$ & 0.95 & 2.84 & $3.669(8)$ & 146 & $-x+1,-y+1, z+1$ \\
\hline & $\mathrm{C}(6)-\mathrm{H}(6 \mathrm{~A}) \cdots \mathrm{Cl}(1)$ & 0.95 & 2.83 & $3.760(9)$ & 168 & $x, y-1, z$ \\
\hline & $\mathrm{C}(2)-\mathrm{H}(2 \mathrm{~B}) \cdots \mathrm{Cl}(2)$ & 0.99 & 2.72 & $3.664(6)$ & 159 & $-x+1, y-1 / 2,-z+1 / 2$ \\
\hline
\end{tabular}

${ }^{\mathrm{a}} \mathrm{X}=\mathrm{Cl} / \mathrm{Br}$.

Table 8 Selected bond lengths and angles in the structure of II.

\begin{tabular}{|c|c|c|c|c|c|c|c|}
\hline Bond & $d, \AA$ & Angle & $\omega, \operatorname{deg}$ & Bond & $d, \AA$ & Angle & $\omega$, deg \\
\hline $\mathrm{Cu}-\mathrm{C}(6)$ & $2.053(4)$ & $\mathrm{C}(6)-\mathrm{Cu}-\mathrm{C}(5)$ & $38.54(16)$ & $\mathrm{N}(2)-\mathrm{C}(7)$ & $1.532(5)$ & $\mathrm{C}(4)-\mathrm{N}(2)-\mathrm{C}(7)$ & $112.5(3)$ \\
\hline $\mathrm{Cu}-\mathrm{C}(5)$ & $2.070(3)$ & $\mathrm{C}(6)-\mathrm{Cu}-\mathrm{X}(1)$ & $111.06(12)$ & $C(1)-C(2)$ & $1.463(5)$ & $\mathrm{N}(1)-\mathrm{C}(1)-\mathrm{C}(2)$ & $178.4(4)$ \\
\hline $\mathrm{Cu}-m^{a}$ & $1.945(5)$ & $\mathrm{C}(5)-\mathrm{Cu}-\mathrm{X}(1)$ & $147.68(11)$ & $C(2)-C(3)$ & $1.527(5)$ & $C(1)-C(2)-C(3)$ & $108.5(3)$ \\
\hline $\mathrm{Cu}-\mathrm{X}(1)^{b}$ & $2.2549(9)$ & $\mathrm{C}(6)-\mathrm{Cu}-\mathrm{X}(2)$ & $139.33(12)$ & $C(4)-C(5)$ & $1.502(5)$ & $\mathrm{N}(2)-\mathrm{C}(3)-\mathrm{C}(2)$ & $111.3(3)$ \\
\hline $\mathrm{Cu}-\mathrm{X}(2)^{b}$ & $2.2853(10)$ & $\mathrm{C}(5)-\mathrm{Cu}-\mathrm{X}(2)$ & $100.96(11)$ & $C(5)=C(6)$ & $1.361(6)$ & $C(6)-C(5)-C(4)$ & $122.6(4)$ \\
\hline $\mathrm{Cu}-\mathrm{X}(2)^{i c}$ & $2.8505(10)$ & $\mathrm{X}(1)-\mathrm{Cu}-\mathrm{X}(2)$ & $107.18(4)$ & $C(7)-C(8)$ & $1.492(5)$ & $\mathrm{C}(6)-\mathrm{C}(5)-\mathrm{Cu}$ & $70.1(2)$ \\
\hline$N(1)-C(1)$ & $1.134(5)$ & $\mathrm{C}(6)-\mathrm{Cu}-\mathrm{X}(2)^{i}$ & $91.62(12)$ & $C(8)=C(9)$ & $1.311(6)$ & $\mathrm{C}(4)-\mathrm{C}(5)-\mathrm{Cu}$ & $111.7(2)$ \\
\hline $\mathrm{N}(2)-\mathrm{C}(3)$ & $1.504(5)$ & $\mathrm{C}(5)-\mathrm{Cu}-\mathrm{X}(2)^{i}$ & $98.13(11)$ & & & $C(5)-C(6)-C u$ & $71.4(2)$ \\
\hline \multirow[t]{4}{*}{$\mathrm{N}(2)-\mathrm{C}(4)$} & $1.511(5)$ & $\mathrm{X}(1)-\mathrm{Cu}-\mathrm{X}(2)^{i}$ & $92.26(3)$ & & & $\mathrm{C}(8)-\mathrm{C}(7)-\mathrm{N}(2)$ & $112.1(3)$ \\
\hline & & $\mathrm{X}(2)-\mathrm{Cu}-\mathrm{X}(2)^{i}$ & $100.30(2)$ & & & $C(9)-C(8)-C(7)$ & $122.3(4)$ \\
\hline & & $\mathrm{C}(3)-\mathrm{N}(2)-\mathrm{C}(4)$ & $111.2(3)$ & & & $\mathrm{C}(5)-\mathrm{C}(4)-\mathrm{N}(2)$ & $113.6(3)$ \\
\hline & & $\mathrm{C}(3)-\mathrm{N}(2)-\mathrm{C}(7)$ & $110.0(3)$ & & & & \\
\hline
\end{tabular}

\footnotetext{
${ }^{\mathrm{a}} m$ - middle point of the $\mathrm{C}(8)=\mathrm{C}(9)$ bond.

${ }^{\mathrm{b}} \mathrm{X}(1) \equiv(0.959(4) \mathrm{Cl}+0.041(4) \mathrm{Br}) ; \mathrm{X}(2) \equiv(0.979(4) \mathrm{Cl}+0.021(4) \mathrm{Br})$.

${ }^{\mathrm{c}}$ Symmetry code: $(i)-x+1, y+1 / 2,-z+1 / 2$.
} 
Table 9 Selected bond lengths and angles in the structure of III.

\begin{tabular}{l|l|l|l|l|l|l|l}
\hline \multicolumn{1}{c|}{ Bond } & \multicolumn{1}{c|}{$d, \AA$} & \multicolumn{1}{c|}{ Angle } & \multicolumn{1}{c|}{$\omega$, deg } & \multicolumn{1}{c|}{ Bond } & \multicolumn{1}{c|}{$d, \AA$} & \multicolumn{1}{c|}{ Angle } & $\omega$, deg \\
\hline $\mathrm{Cu}-\mathrm{N}(1)$ & $2.049(4)$ & $\mathrm{N}(1)-\mathrm{Cu}-\mathrm{Cl}(2)$ & $118.22(16)$ & $\mathrm{C}(3)-\mathrm{N}(2)$ & $1.488(4)$ & $\mathrm{N}(2)-\mathrm{C}(4)-\mathrm{C}(5)$ & $112.1(3)$ \\
$\mathrm{Cu}-\mathrm{N}(11)$ & $1.727(6)$ & $\mathrm{N}(11)-\mathrm{Cu}-\mathrm{Cl}(2)$ & $125.8(3)$ & $\mathrm{N}(2)-\mathrm{C}(4)$ & $1.491(4)$ & $\mathrm{N}(1)-\mathrm{C}(1)-\mathrm{C}(2)$ & $176.6(4)$ \\
$\mathrm{Cu}-\mathrm{Cl}(2)$ & $2.2952(8)$ & $\mathrm{Cl}(2)-\mathrm{Cu}-\mathrm{Cl}(3)^{i}$ & $104.7(2)$ & $\mathrm{C}(4)-\mathrm{C}(5)$ & $1.503(3)$ & $\mathrm{C}(1)-\mathrm{C}(2)-\mathrm{C}(3)$ & $111.7(3)$ \\
$\mathrm{Cu}-\mathrm{Cl}(3)^{i} a$ & $2.301(5)$ & $\mathrm{N}(1)-\mathrm{Cu}-\mathrm{Cl}(3)^{i}$ & $99.2(3)$ & $\mathrm{C}(5)-\mathrm{O}(1)$ & 1.4153 & $\mathrm{~N}(2)-\mathrm{C}(3)-\mathrm{C}(2)$ & $108.8(2)$ \\
$\mathrm{Cu}-\mathrm{Cl}(1)$ & $2.3949(8)$ & $\mathrm{N}(11)-\mathrm{Cu}-\mathrm{Cl}(3)^{i}$ & $101.3(4)$ & $\mathrm{N}(11)-\mathrm{C}(11)$ & $1.141(5)$ & $\mathrm{O}(1)-\mathrm{C}(5)-\mathrm{C}(4)$ & $111.46(15)$ \\
$\mathrm{Cu}-\mathrm{Cl}(3)$ & $2.473(5)$ & $\mathrm{N}(1)-\mathrm{Cu}-\mathrm{Cl}(1)$ & $117.34(12)$ & $\mathrm{C}(11)-\mathrm{C}(21)$ & $1.466(5)$ & $\mathrm{C}(11)-\mathrm{N}(11)-\mathrm{Cu}$ & $176.4(8)$ \\
$\mathrm{Cu}-\mathrm{Cl}(31)$ & $2.470(11)$ & $\mathrm{N}(11)-\mathrm{Cu}-\mathrm{Cl}(1)$ & $108.2(3)$ & $\mathrm{C}(21)-\mathrm{C}(31)$ & $1.518(5)$ & $\mathrm{N}(11)-\mathrm{C}(11)-\mathrm{C}(21)$ & $179.2(8)$ \\
$\mathrm{Cl}(3)-\mathrm{Cl}(3)^{\mathrm{i}}$ & $0.68(3)$ & $\mathrm{Cl}(2)-\mathrm{Cu}-\mathrm{Cl}(1)$ & $103.06(2)$ & $\mathrm{C}(31)-\mathrm{N}(21)$ & $1.481(5)$ & $\mathrm{C}(11)-\mathrm{C}(21)-\mathrm{C}(31)$ & $111.1(4)$ \\
$\mathrm{Cl}(31)-\mathrm{Cl}(3)^{i}$ & $0.444(5)$ & $\mathrm{Cl}(3)-\mathrm{Cu}-\mathrm{Cl}(1)$ & $114.1(4)$ & $\mathrm{N}(21)-\mathrm{C}(41)$ & $1.495(5)$ & $\mathrm{N}(21)-\mathrm{C}(31)-\mathrm{C}(21)$ & $110.4(4)$ \\
$\mathrm{N}(1)-\mathrm{C}(1)$ & $1.141(4)$ & $\mathrm{N}(11)-\mathrm{Cu}-\mathrm{Cl}(31)$ & $100.6(6)$ & $\mathrm{C}(41)-\mathrm{C}(51)$ & $1.505(5)$ & $\mathrm{C}(31)-\mathrm{N}(21)-\mathrm{C}(41)$ & $114.0(4)$ \\
$\mathrm{C}(1)-\mathrm{C}(2)$ & $1.464(4)$ & $\mathrm{N}(1)-\mathrm{Cu}-\mathrm{Cl}(31)$ & $100.1(5)$ & $\mathrm{C}(51)-\mathrm{O}(11)$ & $1.417(4)$ & $\mathrm{N}(21)-\mathrm{C}(41)-\mathrm{C}(51)$ & $111.0(4)$ \\
$\mathrm{C}(2)-\mathrm{C}(3)$ & $1.521(4)$ & $\mathrm{C}(1)-\mathrm{N}(1)-\mathrm{Cu}$ & $162.2(4)$ & & & $\mathrm{O}(11)-\mathrm{C}(51)-\mathrm{C}(41)$ & $108.2(4)$ \\
\hline
\end{tabular}

${ }^{\mathrm{a}}$ Symmetry codes: $(\mathrm{i})-x+1, y,-z+1 / 2,(\mathrm{ii})-x, y,-z+1 / 2$.

Table 10 Selected bond lengths and angles in the structures of IV.

\begin{tabular}{l|l|l|l|l|l|l|l}
\hline \multicolumn{1}{c|}{ Bond } & \multicolumn{1}{c|}{$d, \AA$} & \multicolumn{1}{c|}{ Angle } & \multicolumn{1}{c|}{$\omega, \mathrm{deg}$} & \multicolumn{1}{c}{ Bond } & \multicolumn{1}{c|}{$d, \AA$} & \multicolumn{1}{c}{ Angle } & \multicolumn{1}{c}{$\omega, \mathrm{deg}$} \\
\hline $\mathrm{Cu}-\mathrm{C}(6)$ & $2.0585(16)$ & $\mathrm{C}(6)-\mathrm{Cu}-\mathrm{C}(5)$ & $38.66(6)$ & $\mathrm{N}(2)-\mathrm{C}(7)$ & $1.5151(13)$ & $\mathrm{C}(4)-\mathrm{N}(2)-\mathrm{C}(7)$ & $113.72(8)$ \\
$\mathrm{Cu}-\mathrm{C}(5)$ & $2.0755(16)$ & $\mathrm{C}(6)-\mathrm{Cu}-\mathrm{Cl}(1)$ & $141.55(5)$ & $\mathrm{N}(2)-\mathrm{C}(3)$ & $1.5019(17)$ & $\mathrm{N}(1)-\mathrm{C}(1)-\mathrm{C}(2)$ & $178.9(4)$ \\
$\mathrm{Cu}-m^{a}$ & $1.9505(5)$ & $\mathrm{C}(5)-\mathrm{Cu}-\mathrm{Cl}(1)$ & $102.90(5)$ & $\mathrm{C}(8)-\mathrm{O}(1)$ & $1.4174(15)$ & $\mathrm{C}(1)-\mathrm{C}(2)-\mathrm{C}(3)$ & $110.33(7)$ \\
$\mathrm{Cu}-\mathrm{Cl}(1)$ & $2.2786(7)$ & $\mathrm{C}(6)-\mathrm{Cu}-\mathrm{Cl}(2)$ & $107.84(5)$ & $\mathrm{C}(2)-\mathrm{C}(3)$ & $1.5353(18)$ & $\mathrm{N}(2)-\mathrm{C}(3)-\mathrm{C}(2)$ & $109.66(11)$ \\
$\mathrm{Cu}-\mathrm{Cl}(2)$ & $2.2715(8)$ & $\mathrm{C}(5)-\mathrm{Cu}-\mathrm{Cl}(2)$ & $144.07(4)$ & $\mathrm{C}(1)-\mathrm{C}(2)$ & $1.459(5)$ & $\mathrm{C}(6)=\mathrm{C}(5)-\mathrm{C}(4)$ & $122.76(14)$ \\
$\mathrm{C}(4)-\mathrm{C}(5)$ & $1.503(2)$ & $\mathrm{Cl}(1)-\mathrm{Cu}-\mathrm{Cl}(2)$ & $108.69(18)$ & $\mathrm{N}(1)-\mathrm{C}(1)$ & $1.140(6)$ & $\mathrm{C}(6)=\mathrm{C}(5)-\mathrm{Cu}$ & $70.00(9)$ \\
$\mathrm{C}(5)=\mathrm{C}(6)$ & $1.369(2)$ & $\mathrm{C}(5)-\mathrm{C}(4)-\mathrm{N}(2)$ & $112.29(11)$ & $\mathrm{C}(7)-\mathrm{C}(8)$ & $1.508(5)$ & $\mathrm{C}(4)-\mathrm{C}(5)-\mathrm{Cu}$ & $110.62(10)$ \\
$\mathrm{N}(2)-\mathrm{C}(4)$ & $1.5191(17)$ & $\mathrm{C}(3)-\mathrm{N}(2)-\mathrm{C}(4)$ & $111.42(9)$ & & & $\mathrm{C}(5)=\mathrm{C}(6)-\mathrm{Cu}$ & $71.34(6)$ \\
& & $\mathrm{C}(3)-\mathrm{N}(2)-\mathrm{C}(7)$ & $112.70(9)$ & & & $\mathrm{C}(8)-\mathrm{C}(7)-\mathrm{N}(2)$ & $115.86(6)$ \\
& & & & & & & \\
\end{tabular}

${ }^{\mathrm{a}} m-$ middle point of the $\mathrm{C}(5)=\mathrm{C}(6)$ bond.

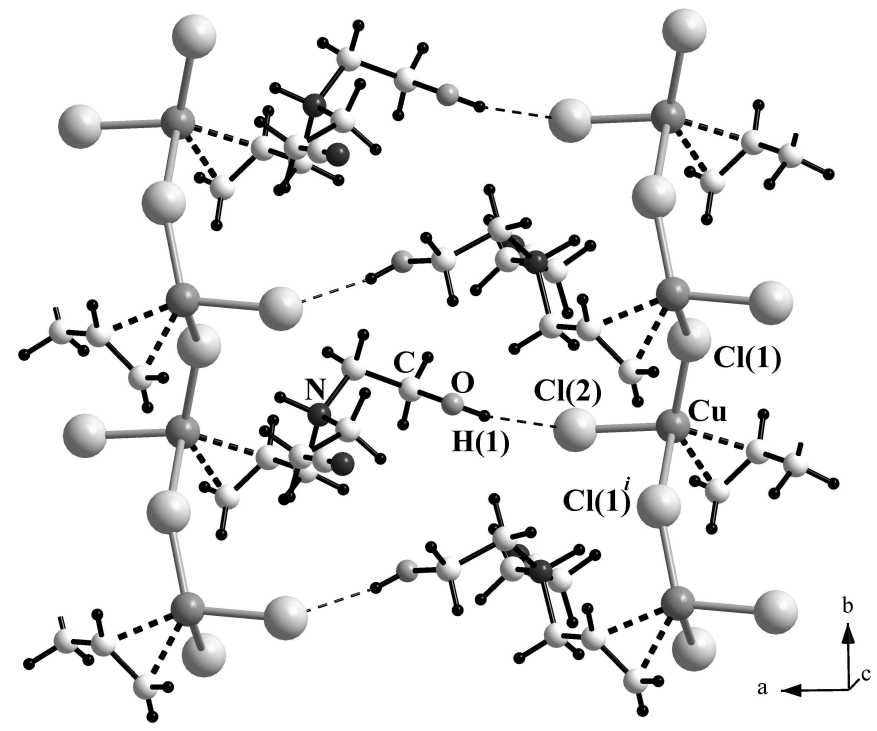

Fig. 6 Projection of the structure of $\left[\left\{\mathrm{HOC}_{2} \mathrm{H}_{4}\left(\mathrm{C}_{3} \mathrm{H}_{5}\right) \mathrm{N}\left(\mathrm{H}^{+}\right) \mathrm{C}_{2} \mathrm{H}_{4} \mathrm{CN}\right\} \mathrm{CuCl}_{2}\right](\mathbf{I V})$.

Symmetry code: (i) $1-x,-1 / 2+y, 1 / 2-z$. 


\section{References}

[1] A. Strecker, Ann. Chem. Pharm. 75 (1850) 27.

[2] D. Enders, J.P. Shilvock, Chem. Soc. Rev. 29 (2000) 359-373.

[3] E.J. Corey, M.J. Grogan, Org. Lett. 1(1) (1999) 157-160.

[4] E.N. Zil'berman, Russ. Chem. Rev. 31(11) (1962) 615-633.

[5] E.N. Zil'berman, Reactions of Nitriles, Khimiya, Moscow, 1972, 448 p. (in Russian).

[6] M.Yu. Luk'yanov, A.V. Pavlyuk, M.G. Mys'kiv, Russ. J. Coord. Chem. 38(2) (2012) 86-91.

[7] J. Houben, Die Methoden der Organischen Chemie, Vol. 4, Thieme Verlag, Leipzig, 1941, $1546 \mathrm{p}$.

[8] B.M. Mykhalichko, M.G. Mys'kiv, Ukr. Patent No. 25450A, Bull. No. 6, 1998.

[9] CrystalClear, Rigaku Corporation, The Woodlands, Texas, 1999.

[10] CrysAlis RED. Version 1.171.31.8 (Release 12_01_2007. CrysAlis 171.NET), Oxford Diffraction Ltd., Oxford, 2007.
[11] R.C. Clark, J.S. Reid, Acta Crystallogr. A 51(6) (1995) 887.

[12] G.M. Sheldrick, SHELXS-97, SHELXL-97, Programs for the Solution and Refinement of Crystal Structures, University of Göttingen, Göttingen, 1997.

[13] DIAMOND v3.1, Crystal Impact GbR, Bonn, 2004-2005.

[14] V.V. Oliinik, M.G. Mys'kiv, L.G. Aksel'rud, Russ. J. Coord. Chem. 22(1) (1996) 71-75.

[15] G.R. Desiraju, T. Steiner, Weak Hydrogen Bonds in Structural Chemistry and Biology, Oxford University Press, Oxford, 1999, 507 p.

[16] D. Braga, F. Grepioni, J. Chem. Soc., Dalton Trans. (1) (1999) 1-8.

[17] G.R. Desiraju, Acc. Chem. Res. 35(7) (2002) 565-573.

[18] M.Yu. Luk'yanov, A.V. Pavlyuk, E.A. Goreshnik, M.G. Mys'kiv. Russ. J. Coord. Chem. 38(9) (2012) 639-645. 